\title{
Random Matrix Theory and the Anderson Model
}

\author{
Jean Bellissard ${ }^{1,3}$ \\ ${ }^{1}$ Georgia Institute of Technology, School of Mathematics, Atlanta GA 30332-0160 \\ ${ }^{3}$ Institut Universitaire de France
}

Dedicated to Elliott Lieb at the occasion of his 70th birthday

\section{The Anderson Model}

This short review is based upon a recent paper in collaboration with J. Magnen and V. Rivasseau [12] proposing a Random Matrix Model as a zeroth order approximation for the Anderson model at small coupling. It was motivated by a previous work by Spencer et al. [15] with a similar aim after the thesis work of Poirot [38, 32, 33]. Part of the present analysis can be found in a recent review paper [10]. Random Matrix Theory (RMT) has been used with success in many problems of Physics and Mathematics, either as a substitute for the $a b$ initio model or as a guideline for statistical properties. However, there is no example so far of such problems in which it has been showed that there is a random matrix approximation justifying the use of RMT. This review intends to show that such a gap may be filled in the Anderson model, thanks to the thesis work of $\mathrm{G}$. Poirot [38].

The Anderson model is given by the following random Hamiltonian acting on $\ell^{2}\left(\mathbb{Z}^{d}\right)$

$$
H_{\omega} \psi(x)=\sum_{y ;|y-x|=1} \psi(y)+V(x) \psi(x) \quad \psi \in \ell^{2}\left(\mathbb{Z}^{d}\right) .
$$

where $\omega=(V(x))_{x \in \mathbb{Z}^{d}}$ is a family of independent identically distributed random variables such that

$$
\langle V(x)\rangle=0 \quad\left\langle V(x)^{2}\right\rangle=W^{2} .
$$

The Anderson conjecture, resulting from previous analysis by Anderson and his collaborators $[1,2]$, asserts that in dimension $d=1,2$ all states are localized for $W>0$, while for 
$d \geq 3$ there is a metal-insulator transition at low disorder in the band center. By localized it is meant here that (i) the spectrum of $H_{\omega}$ is pure-point with probability one and (ii) the localization length is finite ${ }^{1}$. By metallic behavior it is meant that (i) the spectrum is absolutely continuous and simple [44] with probability one, (ii) the diffusion exponent is $1 / 2$ and (iii) the zero temperature conductivity is nonzero and finite.

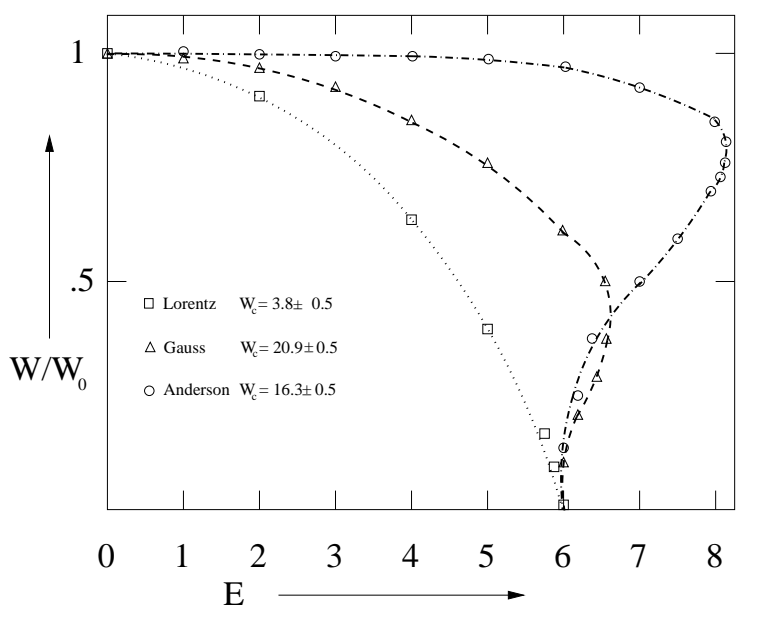

Figure 1: The phase diagram of the 3D Anderson model [29]

The phase diagram in Fig 1 shows the mobility edge in the energy-disorder coordinates $(E, W)$ for the Anderson model with $d=3$ for three types of on-site distribution for the $V(x)$ 's, the uniform, the Gaussian and the Lorentzian distributions. This is the result of years of numerical calculations performed in the eighties [29]. It is interesting to note the existence of a re-entrance phenomenon for the uniform and the Gaussian distributions, namely starting from a low disorder and a fixed energy close to but outside the free spectrum, as $W$ increases, the localized phase is first met then a transition to the extended phase and then again the localized phase.

Historically the previous evidences have been obtained in many ways. Analytic non rigorous works go back to the seminal papers by Anderson et. al. [1, 2]. In 1979, Wegner proposed the $n$-orbital model as a good intuitive model [46]. Namely the wave function $\psi$ in eq.(1) takes on values $\psi(x) \in \mathbb{C}^{n}$ instead, while $V(x)$ is chosen to be an $n \times n$ random matrix of the Gaussian Orthogonal Ensemble (GOE) [34]. He also discussed the existence of a massless Goldstone mode [41] as a sign for delocalization [41]. Numerical works went to supplement these ideas (see [29] and references therein). Rigorous works appeared in the late seventies $[21,30]$ proving that the spectrum of the one-dimensional Anderson model was almost surely pure-point with exponentially localized eigenstates.

\footnotetext{
${ }^{1}$ Mathematical physicists are usually considering only the condition (i) whereas physicist consider usually (ii) only as a definition of localization
} 
In the early eighties these results were extended to higher dimension at large disorder by many contributors starting with the work of Fröhlich and Spencer [18] (see [37] for a list of references). Conceptual improvements were given by Aizenman and Molchanov $[3,4]$ leading to better quantitative estimates on the mobility edge. Very recently, the multiscale analysis proposed by Fröhlich and Spencer was pushed to the limit by Klein and Germinet [20] giving exact and rigorous criterions for the localized phase.

By contrast, the extended phase region still escape rigorous analysis if we except the result announced by Kunz and Souillard and proved by Klein [28] for the Anderson model on a Bethe tree. However the introduction of supersymmetric methods by Wegner and Efetov in the eighties (see [16]) allowed to get some non rigorous important guesses. Eventually, it was realized that RMT could be a good substitute to graph calculations [5] and became the basic tool for calculation in mesoscopic systems (see for instance [6, 26]). Fore indeed a numerical analysis of spectral statistics of the Anderson model for sample sizes smaller than the localization length exhibits a Wigner-Dyson GOE distribution [47]. This is a sign that, at small energy scale at least and on a length scale of the order of the coherent length $\simeq O\left(W^{-2}\right)$, the Anderson Hamiltonian behaves like a random GOE matrix.

It is interesting to notice that such a universal behavior also occurs in non random aperiodic models such as the tight-binding approximation on a quasicrystal [42]. Actually results from localization theory can be used to explain the transport properties of quasicrystalline alloys [39]. It has been argued [10] that RMT dominates in quasicrystals at low temperature whenever the diffusion exponent is larger than $1 / d$.

\section{Transport: Mathematical Background}

In order to define the basic object involved in transport theory, it is convenient to use the formalism of Noncommutative Calculus [14] applied for aperiodic solids firstly in [7, 8, 9] (see [11] for a recent review and references). Let $(\Omega, \mathbb{P})$ be a probability space space on which $\mathbb{Z}^{d}$ acts ergodically by bi-measurable isomorphisms leaving $\mathbb{P}$ invariant. A covariant operator is a family $A=\left(A_{\omega}\right)_{\omega \in \Omega}$ of bounded operators on $\mathfrak{H}=\ell^{2}\left(\mathbb{Z}^{d}\right)$ such that

1. The map $\omega \in \Omega \mapsto A_{\omega} \in \mathcal{B}(\mathfrak{H})$ is measurable.

2. If $T(a)$ denotes the unitary operator of translation by $a$ then $T(a) A_{\omega} T(a)^{-1}=A_{\mathrm{T}^{a} \omega}$ if $\mathrm{T}^{a}$ is the action of $a \in \mathbb{Z}^{d}$ on $\Omega$.

The Anderson Hamiltonian $H$ is an example of such covariant operators whenever the distribution of the $V(x)^{\text {'s }}$ has a compact support. If $H$ is unbounded but almost surely selfadjoint, it is enough to demand that the field of resolvent $\omega \in \Omega \mapsto\left(z \mathbf{1}-H_{\omega}\right)^{-1} \in \mathcal{B}(\mathfrak{H})$ be measurable. The set of such operators is a Von Neumann algebra ${ }^{2}$ if endowed with

\footnotetext{
${ }^{2} \mathrm{~A}$ Von Neumann algebra is a $C^{*}$-algebra with a predual [40]
} 
the pointwise algebraic operations (sum, product, adjoint) and with the norm $\|A\|=$ $\mathbb{P}-\operatorname{esssup}_{\omega \in \Omega}\left\|A_{\omega}\right\|[14]$. An unbounded selfadjoint covariant operator is affiliated to this algebra ${ }^{3}$. There is also a canonical trace given by $[14,11]$

$$
\mathcal{T}_{\mathbb{P}}(A)=\int_{\Omega} d \mathbb{P}(\omega)\left\langle 0\left|A_{\omega}\right| 0\right\rangle=\lim _{\Lambda \uparrow \mathbb{Z}^{d}} \frac{1}{|\Lambda|} \operatorname{Tr}_{\Lambda}\left(A_{\omega}\right) \quad(\mathbb{P} \text { a.e. })
$$

This algebra is denoted $L^{\infty}\left(\mathcal{T}_{\mathbb{P}}\right)^{4}$. Let $\vec{R}=\left(R_{1}, \cdots, R_{d}\right)$ be the position operator acting on $\mathfrak{H}$. Then a derivation $\vec{\nabla}$ is defined by

$$
\vec{\nabla}=\left(\partial_{1}, \cdots, \partial_{d}\right) \quad\left(\partial_{\mu} A\right)_{\omega}=\imath\left[R_{\mu}, A_{\omega}\right]
$$

on the dense subalgebra of covariant operators so that the $\left(\partial_{\mu} A\right)_{\omega}$ 's are bounded almost surely. The Integrated Density of States (IDS) has been proved to satisfy the Shubin formula (see $[8,9])$

$$
\mathcal{N}(E)=\lim _{\Lambda \uparrow \mathbb{Z}^{d}} \frac{1}{|\Lambda|} \#\left\{\text { eigenvalues of } H_{\omega} \Upsilon_{\Lambda} \leq E\right\}=\mathcal{T}_{\mathbb{P}}(\chi(H \leq E)) \quad \text { a.e. } \omega
$$

where $\chi(H \leq E)$ denotes the spectral projection of the covariant Hamiltonian $H$ on the interval $(-\infty, E]$.

Transport properties are expressed through the current-current correlation function defined as the positive matrix valued measure on $\mathbb{R}^{2}$ given by

$$
\mathcal{T}_{\mathbb{P}}\left(f(H) \partial_{\nu} H g(H) \partial_{\nu^{\prime}} H\right)=\int_{\mathbb{R} \times \mathbb{R}} m_{\nu, \nu^{\prime}}\left(d E, d E^{\prime}\right) f(E) g\left(E^{\prime}\right)
$$

for $f, g \in \mathcal{C}_{0}(\mathbb{R})$. Since the electric current operator is given by $\vec{J}=e / \hbar \vec{\nabla} H$, the measure $m$ is formally given by $m_{\nu, \nu^{\prime}}\left(d E, d E^{\prime}\right)=\left\langle E\left|J_{\nu}\right| E^{\prime}\right\rangle\left\langle E^{\prime}\left|J_{\nu^{\prime}}\right| E\right\rangle d E d E^{\prime}$ (up to a multiplicative constant) in terms of the matrix elements of the current in the eigenbasis of the Hamiltonian.

The diffusion exponents are defined so that $L(t) \sim t^{\beta}$ if $L(t)$ is the distance a typical wave packet goes after time $t$. More precisely let $\Delta$ be a Borel subset of $\mathbb{R}$. Let then $\Pi_{\Delta}=\chi(H \in \Delta)$ be the spectral projection of $H$ corresponding to $\Delta$. Then the exponent $\beta_{2}(\Delta)$ is defined by ${ }^{5}$

$$
\left(L_{\Delta}(t)\right)^{2}=\int_{-t}^{+t} \frac{d s}{2 t} \int_{\Omega} d \mathbb{P}(\omega)\left\langle 0\left|\Pi_{\omega, \Delta}\right| \vec{X}_{\omega}(s)-\left.\vec{X}\right|^{2} \Pi_{\omega, \Delta} \mid 0\right\rangle \stackrel{t \uparrow \infty}{\sim} t^{2 \beta_{2}(\Delta)}
$$

\footnotetext{
${ }^{3}$ Namely all its spectral projections on bounded Borel subsets of $\mathbb{R}$ belong to this algebra.

${ }^{4}$ For an intrinsic definition without the help of $\mathfrak{H}$, see $[14,9]$

${ }^{5}$ This is not the only possible definition of the diffusion exponent: see $[22,45,13,23,24,31,43]$ for more details.
} 
Here $f(x) \sim x^{\beta}$ means that $\beta$ is the smallest $\alpha \geq 0$ such that $\int^{+\infty} f(x) d x / x^{1+\alpha}$ converges. It has been shown that, equivalently [43]

$$
m\left\{\left(E, E^{\prime} \in \Delta \times \mathbb{R} ;\left|E-E^{\prime}\right| \leq \epsilon\right\} \stackrel{\epsilon \downarrow 0}{\sim} \epsilon^{2\left(1-\beta_{2}(\Delta)\right)}\right.
$$

(with a similar definition of the exponent). The theory of dissipative transport then shows that, at least in the Relaxation Time Approximation (RTA), the electric conductivity tensor is given by the Kubo formula (see [10] for instance)

$$
\sigma_{\nu, \nu^{\prime}}=\frac{e^{2}}{\hbar} \int_{\mathbb{R}^{2}} m_{\nu, \nu^{\prime}}\left(d E, d E^{\prime}\right) \frac{f_{T, \mu}(E)-f_{T, \mu}\left(E^{\prime}\right)}{E^{\prime}-E} \frac{1}{\hbar / \tau_{\text {coll }}-\imath\left(E^{\prime}-E\right)},
$$

if $T$ denotes the temperature, $\mu$ the chemical potential, $\tau_{\text {coll }}$ the average collision time and $k_{B}$ the Boltzmann constant. In addition $f_{T, \mu}(E)=\left(1+e^{(E-\mu) / k_{B} T}\right)^{-1}$ denotes the Fermi-Dirac distribution. The chemical potential is determined by $\int \mathcal{N}(d E) f_{T, \mu}(E)=n_{e l}$ in terms of the charge carrier density density $n_{e l}$. The Fermi energy $E_{F}$ is the limit of the chemical potential at zero temperature for a fixed $n_{e l}$. Then it has been proved (see [10] for instance)

Theorem 1 If $m_{\nu, \nu^{\prime}}=\rho_{\nu, \nu^{\prime}}^{(2)} d E d E^{\prime}$ with $\rho_{\nu, \nu^{\prime}}^{(2)}\left(E, E^{\prime}\right)$ continuous near $E=E^{\prime}=E_{F}$ then, for any Borel set $\Delta \subset \mathbb{R}$ small enough containing $E_{F}$

1. $\beta_{2}(\Delta)=1 / 2$

2. The diffusion constant $D(\Delta)=\lim _{t \uparrow \infty} L_{\Delta}(t)^{2} / t$ is finite and

$$
D(\Delta)=\pi \int_{\Delta} d E \sum_{\nu} \rho_{\nu, \nu}^{(2)}(E, E)
$$

3. The longitudinal DC conductivity at zero temperature is finite and given by

$$
\sigma_{\nu, \nu}=\frac{\pi e^{2}}{\hbar} \rho_{\nu, \nu}^{(2)}\left(E_{F}, E_{F}\right)
$$

Hence, a sufficient condition for the Anderson model to have a metallic phase is that its current-current correlation function be continuous in the previous sense near the Fermi energy. It ought to be remarked that because of the cubic symmetry of the Anderson model, the conductivity tensor is proportional to the identity matrix (isotropy). 


\section{Random Matrix Models}

\section{$3.1 \quad$ Freeness}

In the theory of random matrices, the Voiculescu free calculus $[48,25]$ describes the combinatorics of moments of a family of independent random $N \times N$ matrices in the limit $N \rightarrow \infty$. More precisely, in this limit these matrices define a unital algebra $\mathcal{A}$ together with a distribution, namely a linear map $\phi: \mathcal{A} \mapsto \mathbb{C}$ with $\phi(\mathbf{1})=1$. This algebra is highly non commutative in that, in this limit, no polynomial relation between the various matrices exists. This is the main idea behind the notion of freeness. Moreover, $\phi$ is defined by averaging products of powers of these matrices (noncommutative moments).

Any element $X \in \mathcal{A}$ is called a random variable. The distribution of $X$ is the map $\phi_{X}: p \in \mathbb{C}[X] \mapsto \phi(p(X)) \in \mathbb{C}$, where $\mathbb{C}[X]$ denotes the set of polynomials in $X$ with coefficients in $\mathbb{C}$. A family $X_{1}, \cdots, X_{n}$ of random variable is called free whenever given any set $p_{1}, \cdots, p_{l}$ of polynomials and any family $\left(i_{1}, \cdots, i_{l}\right)$ of integers in $[1, n]$ such that (i) $i_{k} \neq i_{k+1}$ for all $k$ 's and (ii) $\phi\left(p_{k}\left(X_{i_{k}}\right)\right)=0$, then $\phi\left(p_{1}\left(X_{i_{1}}\right) \cdots p_{l}\left(X_{i_{l}}\right)\right)=0$. It is tedious but straightforward to check that knowing the distribution of each of the $X_{i}$ 's gives all the noncommutative moments uniquely. Hence the knowledge of the marginal and freeness determine uniquely the distribution $\phi$. In particular, given a free pair $X, Y$ of random variables with distribution $\phi_{X}$ and $\phi_{Y}$ respectively, the distribution of $X+Y$ defines in a unique way a free convolution $\phi_{X+Y}=\phi_{X} \boxplus \phi_{Y}$. Voiculescu then discovered the free analog of the Fourier transform that he called the $R$-transform. Let $G_{X}(z)$ be the Green function associated with $X$, namely $G_{X}(z)=\phi\left((z-X)^{-1}\right.$ ) (as a formal power series in $1 / z)$. Then the $R$-transform of $X$ is the formal power series $R_{X}(u)$ defined through

$$
G_{X}=\frac{1}{z-R_{X} \circ G_{X}(z)}
$$

$R_{X}(u)$ is the free analog of the cumulant expansion. In particular

Theorem 2 ([48]) If $X, Y$ are free, then

$$
R_{X+Y}=R_{X}+R_{Y}
$$

In pratical cases, the distribution of a random variable can be extended as a positive measure on $\mathbb{R}$. Among the possible distributions is the semicircular law, given by

$$
\phi_{X}(p)=\frac{2}{\pi} \int_{-1}^{1} d x \sqrt{1-x^{2}} p(x)
$$

Wigner showed that this is the Density of States (DOS) for the various Gaussian ensembles [34]. This is actually also a consequence of the following free central limit theorem [48] 
Theorem 3 (Free Central Limit Theorem) Let $\left(X_{n}\right)_{n \in \mathbb{N}}$ be a countable family of $i$ dentically distributed free random variables with mean zero and variance 1 . Then the limiting distribution of $Y_{n}=n^{-1 / 2} \sum_{i=1}^{n} X_{i}$ is a semicircular law.

The proof is made elementary by using the $R$-transform.

\subsection{Gaussian Random Hamiltonian}

In a physical situation where the electronic Hamiltonian $H$ behaves like a random matrix of one of the Gaussian ensembles, it is expected that the eigenbasis of $H$ is maximally random, namely it can be chosen by random, uniformly with respect to the Haar measure of the orthogonal (or unitary) group. Therefore the Hamiltonian should not determine a preferred orthogonal basis. On the other hand, the current operator $\vec{J}=\imath[\vec{R}, H]$ is usually deterministic since the position operator commutes with the potential. In particular its eigenbasis is well determined. Therefore it is expected that the pair $\vec{J}, H$ be free. If so, using the freeness relation

$$
\phi(X Y Z Y)=\phi(X) \phi\left(Y^{2}\right) \phi(Z)
$$

valid if $\phi(Y)=0$, leads to

$$
\mathcal{T}_{\mathbb{P}}(f(H) \vec{\nabla} H g(H) \vec{\nabla} H)=\mathcal{T}_{\mathbb{P}}(f(H)) \mathcal{T}_{\mathbb{P}}(g(H)) \mathcal{T}_{\mathbb{P}}\left(\vec{\nabla} H^{2}\right)
$$

so that the current-current correlation becomes

$$
m\left(d E, d E^{\prime}\right)=\mathcal{T}_{\mathbb{P}}\left(\vec{\nabla} H^{2}\right) \mathcal{N}(d E) \mathcal{N}\left(d E^{\prime}\right)
$$

In particular if the DOS is given by a semicircular laws, the hypothesis of Theorem 1 are satisfied and this model has a metallic behaviour. In practice, however, $H$ and $\vec{\nabla} H$ are not exactly free so that extra work is needed to conclude.

\subsection{The $n$-orbital Model}

The next example is provided by the Wegner $n$-orbital model. It acts on the Hilbert space $\mathfrak{H}=\ell^{2}\left(\mathbb{Z}^{d}\right) \otimes \mathbb{C}^{n}$ as

$$
H_{\omega} \psi(x)=\sum_{y ;|y-x|=1} \psi(y)+V(x) \psi(x) \quad \psi \in \ell^{2}\left(\mathbb{Z}^{d}\right) \otimes \mathbb{C}^{n}
$$

where now $\omega=\{V(x)\}_{x \in \mathbb{Z}^{d}}$ is a family of $n \times n$ identically distributed independent Gaussian random selfadjoint matrices such that

$$
\langle V(x)\rangle=0 \quad\left\langle(V(x))_{i, j}^{2}\right\rangle=\frac{W^{2}}{n}
$$

As a consequence the following theorem has been proved $[46,27,36]$ 
Theorem 4 1. In the limit $n \rightarrow \infty$ the $V(x)$ 's and the free lattice Laplacean become free variables.

2. In the limit $n \rightarrow \infty$ the density of states and the current-current correlation of the Wegner model are continuous.

3. In this limit the n-orbital model is equivalent to the Random Phase Approximation (RPA) of the Anderson model.

Hence this model is metallic at any disorder.

\subsection{The DPS Model}

In a rigorous paper, Disertori et al. [15] proposed a random matrix model acting on $\mathbb{Z}^{3}$ liable to be much closer to the $3 D$ Anderson model than the previous examples. Here $H=\left(H_{i j}\right)$ is a random gaussian matrix with zero average and covariance

$$
\left\langle H_{i j} H_{k l}\right\rangle=\delta_{i j} \delta_{k l} J_{i j} \quad J_{i j}=\left(\frac{1}{-W^{2} \Delta+1}\right)_{i j}
$$

where $i, j$ vary in $\Lambda \cap \mathbb{Z}^{3}, \Lambda$ a union of cubes of size $W$ and $W>0$ large but fixed. $\Delta$ is the discrete Laplacian with periodic b.c.. As usual the DOS $\rho_{\Lambda}=d \mathcal{N} / d E$ can be obtained through

$$
\rho_{\Lambda}(E)=\frac{1}{\pi} \lim _{\epsilon \downarrow 0} \Im\left\langle\left(\frac{1}{E+\imath \epsilon-H}\right)_{00}\right\rangle
$$

The derivative of the DOS is the imaginary part of $\sum_{x} R\left(E+\imath 0^{+} ; 0, x\right) / \pi$ where $R(E+$ $\imath \epsilon ; 0, x)$ is defined by

$$
\left\langle\left(\frac{1}{E+\imath \epsilon-H}\right)_{0 x}\left(\frac{1}{E+\imath \epsilon-H}\right)_{x 0}\right\rangle
$$

Theorem 5 ([15]) For $W$ large enough the DOS of this model is smooth and coincides, in $[-2,2]$, with the Wigner semicircular distribution modulo a correction of order $W^{-2}$. Moreover $R(E+\imath \epsilon ; 0, x)$ decays exponentially fast in $x$ uniformly as $\epsilon \downarrow 0$ and as $\Lambda \uparrow \mathbb{Z}^{3}$.

\section{The Flip Matrix Model}

\subsection{From Anderson to Wegner}

In his Ph. D. Thesis, G. Poirot developed an idea coming form the field theory analysis of the Anderson model, namely he showed that the Anderson Hamiltonian can be analyzed, 
at small disorder, through a random matrix model called flip matrix [38]. The idea goes roughly as follows. At very small disorder, the dominant term is the discrete Laplacean, denoted by $H_{0}$. Since only the neighborhood of the Fermi energy is relevant for the electronic transport, it is reasonable to reduce the Hamiltonian to a small strip near $E_{F}$. Actually, since the potential has zero average, the lowest order term in perturbation theory for the energy is $O\left(W^{2}\right)$ so that energies $E$ with $\left|E-E_{F}\right| \geq O\left(W^{2}\right)$ can be treated in perturbation theory. For this reason, let $\chi_{W}$ be the spectral projection of $H_{0}$ on the energies $E$ such that $\left|E-E_{F}\right| \leq c \cdot W^{2}$ for some $c$ large enough but independent of $W$. Since $H_{0}$ is translation invariant, its spectral analysis can be performed through Fourier's transform in the quasimomentum space, namely $H_{0}$ is the multiplication by a band function $E(k)$ which is periodic in $k \in \mathbb{R}^{d}$ with period group given by the reciprocal lattice (here $2 \pi \mathbb{Z}^{d}$ ). The Fermi surface is then given by the set of $k$ 's for which $E(k)=E_{F}$. Near the band edges of the spectrum of $H_{0}$, the Fermi surface is a sphere, whereas for energies near the band center it can be more involved. In particular the spectrum of $H_{0}$ is highly degenerated. Consequently, at small disorder, a degenerate perturbation theory must be apply. The lowest order contribution is therefore given by the operator $H_{e f f}=\chi_{W} H \chi_{W} \simeq E_{F} \mathbf{1}+\chi_{W} V \chi_{W}$.

If $\chi_{W}$ is actually replaced by a smooth cut-off function of $H_{0}$ localized within $O\left(W^{2}\right)$ from the Fermi surface, its matrix element decay in the space over a distance of order $O\left(W^{-2}\right)$. Therefore beyond this distance the eigenstates of $H_{\text {eff }}$ are likely to be stochastically independent. For this reason let $\mathbb{Z}^{d}$ be divided into cubes of size $O\left(W^{-2}\right)$. Given such a cube $\Lambda$, let $V_{\Lambda}$ be the restriction of $H_{\text {eff }}$ to $\Lambda$. Since the contribution of $H_{0}$ to $H_{\text {eff }}$ is essentially a scalar, $V_{\Lambda}$ is a random matrix of size $n=O\left(W^{-2(d-1)}\right)$. Then the Anderson Hamiltonian can be seen as a tight binding model indexed by the $\Lambda$ 's with a potential $V_{\Lambda}$ and coupled by a translation invariant nearest neighbor operator. In other words, this transformation gives the Anderson model the structure of an $n$-orbital model. However the potential does not belong to a classical Gaussian ensemble!

This can be seen as follows (see Fig 2): the reduction to a cube, with finite volume, is equivalent to discretize the momentum space with typical spacing $O\left(W^{2}\right)$. Therefore the discretized Fermi strip $\Sigma_{F}$ defined by $\chi_{W}$ contains only $n$ points. Then $V_{\Lambda}$ can be seen as an $n \times n$ selfadjoint matrix indexed by $\Sigma_{F}$ and given by

$$
\left\langle k\left|V_{\Lambda}\right| k^{\prime}\right\rangle=\tilde{V}\left(k^{\prime}-k\right)
$$

where $\tilde{V}$ represents the Fourier transform of the sequence $\omega=(V(x))_{x \in \mathbb{Z}^{d}}$. It is not difficult to check that, if the potential is Gaussian, the $\tilde{V}\left(k^{\prime}-k\right)$ 's are independent Gaussian Variables with zero mean and variance approximately equal to $W$. However this variable are equal for equal values of the vector $k^{\prime}-k$ (momentum conservation). Even if $k$ and $k^{\prime}$ are submitted to stay on the Fermi surface, there are several pairs $\left(k, k^{\prime}\right) \in \Sigma_{F}^{\times 2}$ with a given value of $k-k^{\prime}$. In dimension $d=2$ there are only two such pairs at most (see Fig 2 ). But for $d \geq 3$ (see Fig. 3 below) the set of such pairs increases with $n$ like $n^{(d-2) /(d-1)}$, so that $V_{\Lambda}$ cannot be in any of the known Gaussian ensembles. 


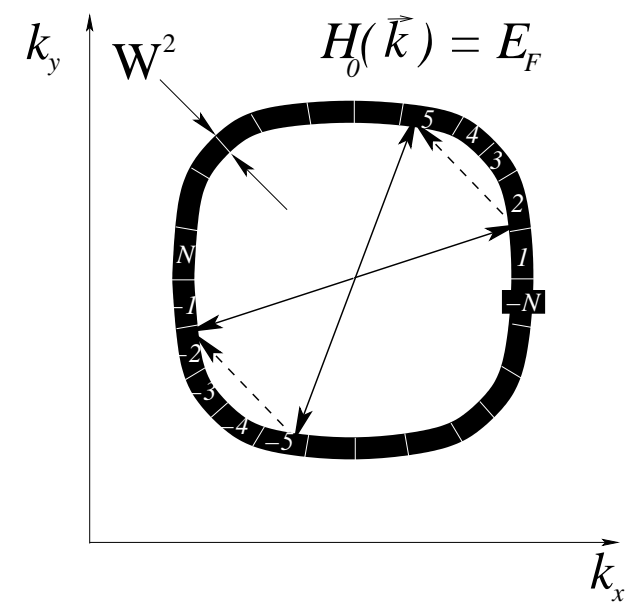

Figure 2: Fermi Surface for the Flip Model

\subsection{Description of the flip model for $d=2$}

Taking advantage of the time reversal symmetry, one can label the points of $\Sigma_{F}$ by indices $\alpha, \beta \in\{1, \cdots, N\} \cup\{-N, \cdots,-1\}$ with $n=2 N$. Namely $k_{-\alpha}=-k_{\alpha}$ (see Fig. 2). Then $V_{\Lambda}$ becomes a selfadjoint random matrix. Together with ther momentum conservation this leads to the following constraints

$$
V_{\alpha, \beta}=\overline{V_{\beta, \alpha}} \quad V_{\alpha, \beta}=V_{-\beta,-\alpha} \quad V_{\alpha, \alpha}=V_{0}
$$

Apart from these constraints, the matrix elements are independent gaussian random variables such that

$$
\left\langle V_{\alpha, \beta}\right\rangle=0 \quad\left\langle\left|V_{\alpha, \beta}\right|^{2}\right\rangle=W^{2}=O(1 / 2 N)
$$

The main result obtained in [12] is the following

Theorem 6 ([12]) The DOS of the flip matrix model is semicircular.

The proof of this result has been obtained through supersymmetric methods [16, 35]. Namely the DOS can be written as supersymmetric integral

$$
\frac{d \mathcal{N}}{d E}=\lim _{\Im E \downarrow 0} \frac{1}{\pi} \int S_{+\alpha}^{+} S_{+\alpha} e^{L} \mathcal{D} \Psi^{\dagger} \mathcal{D} \Psi
$$

where $\Psi_{ \pm \alpha}=\left(S_{ \pm \alpha}, \chi_{ \pm \alpha}\right)$ is a superfield with bosonic part $S_{ \pm \alpha}$ and fermionic part $\left.\chi_{ \pm \alpha}\right)$. Moreover, $L$ is a sum of quartic terms of the form $\Psi_{ \pm \alpha}^{\dagger} \Psi_{ \pm \beta} \Psi_{ \pm \beta}^{\dagger} \Psi_{ \pm \alpha}$. Using the commutation rules, $L$ can be written as a sum of terms of the form $\Psi_{ \pm \alpha}^{\dagger} \Psi_{ \pm \alpha} \Psi_{ \pm \beta}^{\dagger} \Psi_{ \pm \beta}$, allowing for a separation between the $\alpha$ 's and the $\beta$ 's. The sum over $\alpha$ of the terms in $\Psi_{ \pm \alpha}^{\dagger} \Psi_{ \pm \alpha}$ 
defines a mean field so that $L$ is a sum of squares of this mean field. Using a Gaussian integral again (Hubbard-Stratonovich transformation), the quartic terms can be undone leading to

$$
e^{L}=\int \mathcal{D} R e^{\imath W \sum_{\alpha} \Psi_{\alpha}^{\dagger} R \Psi_{\alpha}}
$$

where $R$ varies in a set of $4 \times 4$ supermatrices. Then the Gaussian integral can be performed. After integrating out the Fermion degrees of freedom, the DOS is given in terms of a 5-dimensional ordinary integral of the form $\int_{\mathbb{R}^{5}} d^{5} x \quad(F(x))^{N}$. Since $N=$ $O\left(W^{-2}\right) \gg 1$, it can be analyzed through a saddle point method. The function $F$ has 4 saddle points, among which only one contributes to the limit $W \downarrow 0$. This latter one gives the semicircular law.

\subsection{Beyond the flip matrix model}
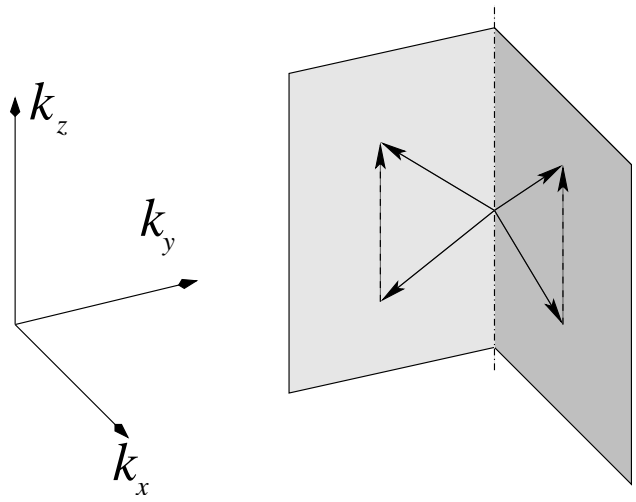

Twisted pairs

Figure 3: Twisted pairs of momenta for $d=3$

For $d \geq 3$ the the degeneracy of the matrix elements in the flip model makes the flip model much more difficult to analyze even with the supersymmetric method. However a power counting of the Feynmann graphs associated with non coplanar pairs $\left(k_{1}, k_{1}^{\prime}\right)$ and $\left(k_{2}, k_{2}^{\prime}\right)$ with $k_{1}^{\prime}-k_{1}=k_{2}^{\prime}-k_{2}$ (see Fig 3) shows that their contribution should be subdominant. If so, only the planar diagram should contribute so that the flip matrix model should again be the dominant contribution. This gives a hope that the previous result also holds in any dimension.

Beside the calculation of the DOS, it will be necessary to compute the current-current correlation function and to show that it is smooth enough so as to apply the Theorem 1. Such calculation can be done through supersymmetry [35]. However, the supersymmetric field has mores degrees of freedom [35] and the zero mode set becomes much more com- 
plicated. Recently Fyodorov [19] has proposed a substitute to the Hubbard-Stratonovich transformation that avoid such a difficult problem.

In any case the previous calculation should be supplemented by an estimate of error terms coming from approximating the Anderson model by the flip one, in a way similar to the analysis of $[38,32,33,15]$.

The supermatrix $R$ found in the previous section can be seen as an effective order parameter for each cube $\Lambda$. The small coupling between cubes should then give rise to nearest neighbor coupling between such parameter, leading to a kind of Ising model with spin given by $R$ or a nonlinear $\sigma$-model [16]. Therefore spin waves are expected at low energy. A long range order will follow if the spin waves are massless: this is the famous Goldstone mode that is predicted by theoretical approaches. It is worth remarking however, that the $R$ matrix was obtained through the calculation of the DOS only. If the current-current correlation function is considered instead, the effective order parameter should be of a different nature. But the existence of a Goldstone mode must also be addressed. In $d=2$, it is expected that the spin wave be massive with a mass $e^{-O\left(W^{-1}\right)}$. This a highly non perturbative result based upon a finite scale renormalization group analysis [2]. To prove that they are massless for $d \geq 3$, one possible method could be the proof of an infrared inequality through Osterwalder-Schrader positivity $[17]^{6}$.

These comments show that there is still a long way to go before a rigorous proof of the existence of a metallic phase in the Anderson model can be established.

Acknowledgements: The author wishes to thank J. Magnen and V. Rivasseau for a year of collaboration in Paris. He wishes to thank the I.H.É.S. and the École Polytechnique for support during the year 2001-2002 while this work was performed. He also wishes to thank Y.V. Fyodorov for bringing [19] to his attention.

\section{References}

[1] P. W. Anderson, "Absence of Diffusion in Certain Random Lattices", Phys. Rev., 109, (1958), 1492-1505.

[2] E. Abrahams, P. W. Anderson, D. C. Licciardello and T. V. Ramakrishnan, "Scaling Theory of Localization: Absence of Quantum Diffusion in Two Dimensions", Phys. Rev. Letters, 42, (1979), 673-676.

[3] M. Aizenman, S. Molchanov, "Localisation at Large Disorder and at Extreme Energies: an Elementary Derivation", Com. Math. Phys., 157, (1993), 245-278.

\footnotetext{
${ }^{6}$ However, O-S positivity may not hold (the author thanks T. Spencer for this remark).
} 
[4] M. Aizenman, "Localisation at Weak Disorder: Some Elementary Bounds", Rev. Math. Phys., 6, (1994), 1163-1182.

[5] B. Altshuler, B. I. Shklovskii, "Repulsion of energy levels and conductivity of small metal samples", Sov. Phys. JETP, 64, (1986), 127-135.

[6] C. W. J. Beenaker, "Random Matrix theory of Quantum Transport",Rev. Mod. Phys., 69, (1997), 731-808.

[7] J. Bellissard, "Schrödinger's operators with an almost periodic potential : an overview" in Lecture Notes in Phys., 153, Springer Verlag, Berlin Heidelberg, New York, (1982).

[8] J. Bellissard, " $K$-Theory of $C^{*}$-algebras in Solid State Physics" in Statistical Mechanics and Field Theory, Mathematical Aspects, T.C. Dorlas, M.N. Hugenholtz \& M. Winnink, Lecture Notes in Physics, 257, (1986), 99-156.

[9] J. Bellissard, "Gap Labelling Theorems for Schrödinger's Operators" in From Number Theory to Physics, pp.538-630, Les Houches March 89, Springer, J.M. Luck, P. Moussa \& M. Waldschmidt Eds., (1993).

[10] J. Bellissard, "Coherent and dissipative transport in aperiodic solids", in Dynamics of Dissipation, Garbaczewski, P.; Olkiewicz, R. (Eds.), Lecture Notes in Physics, 597, (2003), 413-486.

[11] J. Bellissard, "The Noncommutative Geometry of Aperiodic Solids", to appear in the Proceedings of the 2001 Summer School of Theoretical Physics, Geometry, Topology and Quantum Field Theory, Villa de Leyva, Colombia, 7-30 July 2001, Kluwer (2003).

[12] J. Bellissard, J. Magnen,and V. Rivasseau, "Supersymmetric Analysis of a Simplified Two Dimensional Anderson Model at Small Disorder", cond-mat/0210524, to appear in Markov Process and related Fields, (2003).

[13] J.-M. Combes, "Connections between quantum dynamics and spectral properties of time-evolution operators", Differential equations with applications to mathematical physics, 59-68, Math. Sci. Engrg., 192, Academic Press, Boston, MA, (1993).

[14] A. Connes, Noncommutative Geometry, Acad. Press., San Diego (1994).

[15] M. Disertori, H. Pinson, T. Spencer, "Density of states for Random Band Matrix", Commun. Math. Phys., 232, (2002), 83-124.

[16] K. B. Efetov, Supersymmetry in Disorder and Chaos, Cambridge University Press, (1997). 
[17] J. Fröhlich, T. Spencer, B. Simon, "Infrared bounds, phase transition and continuous symmetry breaking", Commun. Math. Phys., 50, (1976), 79-94.

[18] J. Fröhlich, F. Martinelli, E. Scoppola and T. Spencer, "Constructive Proof of Localization in the Anderson tight binding model", Commun. Math. Phys., 101, (1985), 21-46.

[19] Y. V. Fyodorov, "Negative moments of charateristic polynomials of random matrices: Ingham-Siegel integral as an alternative to Hubbard-Stratonovich transformation", Nucl. Phys., B621, (2002), 643-674.

[20] F. Germinet, A. Klein, "Bootstrap multiscale analysis and localization in random media", Commun. Math. Phys., 222, (2001), 415-448.

[21] I. Gold'sheid, S. Molchanov and L. Pastur, "A random homogeneous Schrödinger operator has a pure point spectrum", Funct. Anal. Appl., 11, (1977), 1-10.

[22] I. Guarneri, "Spectral properties of quantum diffusion on discrete lattices", Europhys. Lett., 10, (1989), 95-100.

[23] I. Guarneri, "On an estimate concerning quantum diffusion in the presence of a fractal spectrum", Europhys. Lett., 21, (1993), 729-733.

[24] I. Guarneri, G. Mantica, "On the asymptotic properties of quantum dynamics in the presence of fractal spectrum", Ann. Inst. H. Poincaré, 61, (1994), 369-379.

[25] F. Hiai, D. Petz, The Semicircle Law, Free Random Variables and Entropy, American Mathematical Society, (2000).

[26] Y. Imry, Introduction to Mesoscopic Physics, Oxford Univ. Press, Oxford (1997).

[27] A. Khorunzhy, L. Pastur, " Limits of infinite interaction radius, dimensionality and the number of components for random operators with off-diagonal randomness", Com. Math. Phys., 153, (1993), 605-646.

[28] A. Klein, "Absolutely continuous spectrum in the Anderson model on the Bethe lattice", Math. Res. Lett., 1, (1994), 399-407; "The Anderson metal-insulator transition on the Bethe lattice", XI International Congress of Mathematical Physics (Paris 1994), 383-391, Internat. Press, Cambridge, MA, (1995).

[29] B. Kramer, A. MacKinnon, "Localization: Theory and Experiments", Rep. Prog. Phys., 56, (1993), 1469-1564.

[30] H. Kunz and B. Souillard, "Sur le spectre des opérateurs aux différences finies aléatoires", Comm. Math. Phys., 78, (1980/81), 201-246. 
[31] Y. Last, "Quantum Dynamics and decomposition of singular continuous spectra", J. Funct. Ana., 142, (1996), 402-445.

[32] J. Magnen, G. Poirot and V. Rivasseau, "The Anderson Model as a Matrix Model", Nucl. Phys. B, 58, (1997), 149-162.

[33] J. Magnen, G. Poirot and V. Rivasseau, "Ward type Identities for the 2d Anderson Model at weak disorder", Journ. Stat. Phys., 93, (1998), 331-358.

[34] M. Mehta, Random Matrices, 2nd Ed., Acad. Press, (1990).

[35] A. D. Mirlin, "Statistics of energy levels and eigenfunctions in disordered and chaotic systems: Supersymmetry approach", Proceedings of the International School of Physics Enrico Fermi, Course CXLIII, Eds. G.Casati, I.Guarneri and U.Smilansky, pp.223-298, IOS Press, Amsterdam, (2000), (see cond-mat/0006421).

[36] P. Neu, R. Speicher, "Rigorous mean field model for CPA: Anderson model with free random variables", J. Stat. Phys. 80, (1995), 1279-1308.

[37] L. Pastur, A. Figotin, Spectra of Random and Almost-Periodic Operators, SpringerVerlag, (1992).

[38] G. Poirot, "Mean Green's function of the Anderson Model at weak disorder with an infrared cutoff", Ann. Inst. Henri Poincaré, 70, (1999), 101-146.

[39] S. Roche, D. Mayou \& G. Trambly de Laissardière, "Electronic transport properties of quasicrystals", J. Math. Phys., 38, (1997), 1794-1822.

[40] S, Sakai, $C^{*}$-algebras and $W^{*}$-algebras, Ergebnisse der Mathematik und ihrer Grenzgebiete, Band 60. Springer-Verlag, New York-Heidelberg, (1971).

[41] L. Schäfer, F.J. Wegner, "Disordered system with n orbitals per site: Lagrange Formulation, Hyperbolic Symmetry, and Goldstone Modes", Z. Phyzik, B38, (1980), 113-126.

[42] M. Schreiber, U. Grimm, R. A. Roemer, J. -X. Zhong, "Energy Levels of Quasiperiodic Hamiltonians, Spectral Unfolding, and Random Matrix Theory", Comp. Phys. Commun., 121-122, (1999), 499-501.

[43] H. Schulz-Baldes, J. Bellissard, "Anomalous transport: a mathematical framework", Rev. Math. Phys., 10, (1998), 1-46.

[44] B. Simon, "Cyclic vectors in the Anderson model", Rev. Math. Phys., 6 (1994), 1183-1185. 
[45] R. Strichartz, "Fourier asymptotics of fractal measures", J. Funct. Anal., 89, (1990), 154-187.

[46] F. Wegner, "Disordered system with $\mathrm{n}$ orbitals per site: $\mathrm{n}=\infty$ limit", Phys. Rev., B 19, (1979), 783-792.

[47] I.Kh. Zharekeshev, M. Batsch, B. Kramer, "Crossover of level statistics between strong and weak localization in two dimensions", Europhys. Lett, 34, (1996), 587592.

[48] D. Voiculescu, K. Dykema, A. Nica, Free random variables, AMS, Providence, Rhode Island, (1992). 